\title{
Focal Muscle Vibration: An Emerging Modality in Stroke Rehabilitation
}

\author{
Jawad Ahmed ${ }^{1 *}$, Farheen Malik and Marium Zafar ${ }^{1}$ \\ ${ }^{1}$ Department of Internal Medicine, Dow University of Health Sciences, Karachi, Pakistan
}

\begin{abstract}
Respected Editor,
Stroke is one of the highest contributors to mortality in South East Asia. Its prevalence in Pakistan is assessed to be $250 / 100,000$ population with 350,000 new cases per annum [1]. Stroke is a leading cause of disability where $20-40 \%$ of stroke survivors are affected by spasticity, a complication of upper motor neurons [2]. Spastic paralysis secondary to stroke leads to functional limitations due to ataxia, gait disturbances, joint contracture, and pain that deteriorates the quality of life. Post-stroke spasticity management requires a mix of pharmacological and certain rehabilitative treatments, of which the prevalent ones in Pakistan are virtual reality, early mobilization, movement therapy induced by force and gait training and non-invasive brain stimulation. For the treatment of spasticity, focal muscle vibration (FMV) is more affordable, user-friendly, with no known adverse effects [3]. Despite its promising results, focal muscle vibration has not yet been introduced in Pakistan probably due to lack of literature.
\end{abstract}

Focal muscle vibration (FMV), an effective neurorehabilitative procedure, requires the focal application of vibration of appropriate frequency (variable according to the muscle) on the muscle belly. This stretches the muscle by producing muscle waves. The propagation of muscle waves results in muscle stretch which induces the tonic vibration reflex via spinal circuits [4]. Tonic vibration reflex is a phenomenon where vibration induces involuntary contraction in vibrated muscle and a relaxation of its antagonist [5].

Effectiveness of FMV was proven by Costantino C, et al. in their randomized control trial (RCT) published in 2017. A total of 32 post-stroke patients with chronic upper limb spasticity were divided into two groups. The experimental group (17 patients) was given low amplitude $(2 \mathrm{~mm})$, high frequency $(300 \mathrm{~Hz})$ vibrations for 30 minutes, thrice a week for a month. The control group received sham therapy. Statistically significant difference existed between two groups in modified ashworth scale (MAS) for shoulder $(p=0.007)$, elbow $(p=0.003)$, and wrist $(p$ $<0.001$ ) [6]. Further promising results were proved in $\mathrm{RCT}$ conducted by $\mathrm{Oh} \mathrm{J}$, et al. which was published in late 2017. They included hemiplegic patients with calf muscle spasticity and applied $80 \mathrm{~Hz}$ vibration with an

\footnotetext{
*Corresponding Author: Jawad Ahmed, MBBS, Department of Internal Medicine, Dow University of Health Sciences, Karachi, Pakistan; Email: jawadahmedd13@gmail.com

Received: November 30, 2019; Revised: December 14, 2019; Accepted: December 20, 2019
} DOI: https://doi.org/10.37184/lnjpc.2707-3521.1.5 amplitude of $0.3 \mathrm{~mm}$. It was found that FMV had an antispastic effect on Gastrocnemius. The angle of ankle dorsiflexion and range of movement of the ankle was increased after the application of FMV [7]. Moreover, the use of FMV was found to reduce the risk of falling in the elderly by improving balance [8].

Studies show that FMV has a promising outcome and it can significantly reduce spasticity which can improve quality of life in stroke patients. Further trials should be carried out in our population as well to develop a better understanding of the potential efficacy of FMV. The rehabilitative physicians should be vigilant of newer techniques and modalities being developed across the world and should aim to initiate the use of new scientifically proven beneficial techniques in their practice.

\section{CONFLICT OF INTEREST}

The authors declare no conflict of interest.

\section{ACKNOWLEDGEMENTS}

Jawad Ahmed and Marium Zafar contributed to the manuscript design. All authors significantly contributed to manuscript preparation. Farheen Malik critically revised the manuscript. All authors have read and approved the final version of the manuscript.

\section{REFERENCES}

1. Wasay M, Khatri IA, Kaul S. Stroke in South Asian countries. Nat Rev Neurol 2014; 10: 135-43.

2. Lim SM, Yoo J, Lee E, et al. Acupuncture for Spasticity after Stroke: A systematic review and meta-analysis of randomized controlled trials. Evid Based Complement Alternat Med 2015; 2015: 870398.

3. Murillo N, Valls-Sole J, Vidal J, Opisso E, Medina J, Kumru H. Focal vibration in neurorehabilitation. Eur $\mathrm{J}$ Phys Rehabil Med 2014; 50: 231-42.

4. Guang H, Ji L, Shi Y. Focal vibration stretches muscle fibers by producing muscle waves. IEEE Trans Neural Syst Rehabil Eng 2018; 26: 839-46.

5. Hagbarth K-E, Eklund G. Tonic vibration reflexes (TVR) in spasticity. Brain Res 1966; 2: 201-3.

6. Costantino C, Galuppo L, Romiti D. Short-term effect of local muscle vibration treatment versus sham therapy on upper limb in chronic post-stroke patients: a randomized controlled trial. Eur J Phys Rehabil Med 2017; 53: 32-40.

7. Oh J, Leigh JH, Seo HG, Oh BM, Sangeun J. The effects of focal muscle vibration to reduce calf muscle spasticity in chronic stroke patients: preliminary study. J Neurol Sci 2017; 381: 1134.

8. Toosizadeh N, Mohler J, Marlinski V. Low intensity vibration of ankle muscles improves balance in elderly persons at high risk of falling. PLoS One 2018; 13: e0194720. 